\title{
Recent advances in resolving kinetic and dynamic processes at the air/water interface using specular neutron reflectometry
}

DOI:

10.1016/j.cocis.2018.06.002

\section{Document Version}

Accepted author manuscript

Link to publication record in Manchester Research Explorer

Citation for published version (APA):

Campbell, R. A. (2018). Recent advances in resolving kinetic and dynamic processes at the air/water interface using specular neutron reflectometry. Current Opinion in Colloid and Interface Science, 37, 49-60.

https://doi.org/10.1016/j.cocis.2018.06.002

Published in:

Current Opinion in Colloid and Interface Science

\section{Citing this paper}

Please note that where the full-text provided on Manchester Research Explorer is the Author Accepted Manuscript or Proof version this may differ from the final Published version. If citing, it is advised that you check and use the publisher's definitive version.

\section{General rights}

Copyright and moral rights for the publications made accessible in the Research Explorer are retained by the authors and/or other copyright owners and it is a condition of accessing publications that users recognise and abide by the legal requirements associated with these rights.

\section{Takedown policy}

If you believe that this document breaches copyright please refer to the University of Manchester's Takedown Procedures [http://man.ac.uk/04Y6Bo] or contact uml.scholarlycommunications@manchester.ac.uk providing relevant details, so we can investigate your claim.

\section{OPEN ACCESS}




\title{
Recent Advances in resolving Kinetic and Dynamic Processes at the Air/Water Interface using Specular Neutron Reflectometry
}

\author{
Richard A. Campbell* \\ Division of Pharmacy and Optometry, University of Manchester, Stopford Building, Oxford Road, \\ Manchester M13 9PT, United Kingdom \\ * corresponding author: +44 161275 7844; richard.campbell@manchester.ac.uk.
}

Highlights

- Kinetic and dynamic studies at the air/water interface are becoming more common

- Fast processes can now be resolved accurately using specular neutron reflectometry

- Clear advances have been made using the powerful FIGARO instrument at the ILL

- Data of monolayers now take just 1 second and binary mixtures take just 2 minutes

- The new capabilities have impacted studies in soft matter, environment and health

\section{Abstract}

This review addresses significant recent advances in kinetic and dynamic studies at the air/water interface using specular neutron reflectometry. A step change in the capabilities to resolve these processes in systems related to soft matter, biophysics, environment and health has been delivered primarily by the FIGARO instrument at the Institut Laue-Langevin. It is now routinely carried out to resolve the surface excess of single-component, deuterated monolayers on the second time scale and the interfacial composition of binary mixtures where only one component is deuterated on the minute time scale. This resource has been exploited extensively in the last few years to provide new insight into the kinetics of oxidation of monolayers targeted from the gas phase and the adsorption of various types of molecules from solution as well as the dynamic molecular interactions of systems under continuous flow and during repeated compression/expansion cycles of a Langmuir trough. A perspective is given on current capabilities and future developments that will result from further developments in instrumentation and sample environment.

\section{Introduction}

Specular neutron reflectometry (NR) is a powerful and versatile technique that provides information about the surface excess, composition and structure of thin films at interfaces [1]. It was first applied to adsorbed and spread monolayers at the air/water interface using the CRISP instrument at the ISIS pulsed neutron source in the 1980s [2]. The technique exploits the sensitivity of neutron scattering to the cross sections of different elements and isotopes, while neutron wavelengths on the Ångstrom-scale are often on the order of the thickness of thin molecular films, which results in interference that can be modelled to provide unique information [3]. Indeed the ability to use specular NR to characterize equivalent chemical interfaces in multiple isotopic contrasts results in the provision of a great depth of quantitative information about the sample. The current capabilities of the technique have been summarised in two recent review articles $[4,5]$.

Most scientific problems are tackled nowadays with multiple experimental and theoretical methods, and each one has its strengths and limitations. Specular NR is no different in this respect, and in spite 
of its great potential to solve problems that cannot be solved using other methods, traditionally the technique has a perception of being big and slow. Beam sizes are on the order of tens of square centimetres and typical measurement times for a high quality, full measurement are up to tens of minutes even on the leading instruments. As such, specular NR studies at the air/water interface are often conducted in combination with other approaches such as surface tensiometry [6] and surface pressure isotherms [7], ellipsometry [8,9], Brewster angle microscopy [10,11], external reflection FTIR spectroscopy (ER-FTIRS) [12,13], X-ray reflectometry [14,15] and molecular dynamics simulations $[16,17]$.

In spite of the limitations in the time resolution of specular NR as a technique, the scientific interest in kinetic and dynamic processes at the air/water interface is widespread. Topics of interest include adsorption kinetics in monolayers involving molecules dissolved in solution such as proteins [18], drugs [19], nanoparticles [20] or DNA [21], reaction kinetics of monolayers with molecules present in a saturated gas phase [22], and the response of monolayers to thermal [23] or photochemical [24] stimuli. Kinetic information on such systems is obtained through the acquisition of time-resolved data. Topics further extend to dynamic studies at the air/water interface where the variable is a parameter other than time, such as surface pressure [25], surface oscillation amplitude and frequency [26] or surface expansion rate [27].

\section{Physical basis}

The principles of the specular NR technique, including its physical basis and how neutron reflectivity profiles are recorded and modelled, have been described in detail elsewhere in the past [1] and very recently [5]. As a result, only a brief descriptive summary is given here that focuses on general points pertinent to recent advances in kinetic and dynamic studies at the air/water interface.

Molecules (or parts of molecules) can be described in terms of the sum of the coherent cross sections of nuclei contained in a given volume. This so-called scattering length density is essentially a measure of the strength of a material to scatter neutrons, and is related to its refractive index. When neutrons are incident at an interface, just like with light, they can be transmitted (i.e. refracted) or reflected. Specular NR is a grazing incidence technique where a collimated beam of neutrons is reflected off a sample at an incidence angle up to only a few degrees. The reflectivity, $R$, is the ratio of the number of neutrons in the reflected beam divided by that in the incidence beam. The latter quantity is measured either using a beam monitor before the sample or by conducting a direct beam (or transmission) measurement on the main detector in the absence of a sample. Neutron reflectivity profiles are a representation of $R$ with respect to the momentum transfer normal to the interface, $Q_{z}=4 \pi \cdot \sin \theta / \lambda$, where $\theta$ is the incidence angle and $\lambda$ is the neutron wavelength.

Reflectivity profiles can be recorded in two distinct ways. A monochromatic reflectometer has a narrow band of wavelengths, data are recorded at many different incidence angles, and each measurement results in one data point in the resulting reflectivity profile. Alternatively, a 'time-offlight' (TOF) reflectometer has a broad band of wavelengths, data are recorded at a small number of incidence angles, and the resulting data are binned (or grouped) at discrete intervals in $Q_{2}$ to result in the desired number of data points to form the reflectivity profile. In this case, the different neutron velocities in a spread of wavelengths mean that a neutron detector after the sample recording in time can be calibrated in $\lambda$. In general, TOF reflectometry is the mode of choice for timeresolved studies as data across a broad $Q_{z}$-range can be acquired simultaneously.

Regardless of the type of reflectometer, either can be situated on a pulsed (or spallation) source or a continuous (or reactor) source. In the latter case, spinning discs with discrete openings called 
choppers are used to create the neutron pulses. The advantage of this approach is that the instrument does not need to accept the pulse frequency and dynamic range of the source, but instead these characteristics can be tailored to the need of individual experiments, albeit with a large loss of neutron flux. For example, in general, higher flux at the expense of looser resolution results from more broadly separated choppers, while higher flux at the expense of broader dynamic range results from restricting the maximum accessible wavelength and spinning the discs faster. Such fine control over the needs of the experiment is not as easily accessible at a spallation source where an instrument is supplied with neutron pulses of a pre-defined nature, although in this case instruments have natural access to time-of-flight information with the possibility to exploit a greater proportion of the available neutron flux.

The depth of information that can be resolved in a specular NR experiment is extensive. Experimental data are fitted usually through application of an optical matrix model of the neutron reflectivity at stratified, homogenous layers normal to the interface, each one of which has a given molecular composition and defined roughness [28]. The parameters that can be refined for each layer are normally its scattering length density, thickness, volume fraction of solvent and interfacial roughness. In this case, the scattering length density of each layer is the sum of the contributions from different components normalised to their respective volume fractions. Within the restrictions of modelling software, constraints can be applied to the parameters in the different layers to respect physical reality, such as to ensure that a monolayer of a surfactant has a ratio of chains to headgroups that is consistent with the appropriate molecular stoichiometry. Fitting of a common model to data recorded in multiple isotopic contrasts can allow one to resolve the structure and composition of the material at the interface by selectively highlighting through deuteration individual components (or parts of components) in successive measurements.

Through isotopic substitution, a mixture of $8.1 \% \mathrm{v} / \mathrm{v} \mathrm{D}_{2} \mathrm{O}$ in $\mathrm{H}_{2} \mathrm{O}$ has a scattering length density equal to that of air, and as such the mixture is referred to as 'air contrast matched water' (ACMW). The molecules present at the air/ACMW interface in any such measurement dominate the specular neutron reflectivity, and the magnitude of the reflectivity depends on a combination of their amounts and scattering length densities. Most of the signal from material present at the interface lies at low $Q_{z}$ values (e.g. $<0.05 \AA^{-1}$ ) due to the $Q_{z}^{4}$ falloff in the Fresnel reflectivity, so these measurements are optimal for resolving the surface excess $\Gamma$. For a single component, $\Gamma=\rho_{\mathrm{i}} \cdot d \cdot \phi_{\mathrm{i}} /$ $b_{\mathrm{i}} \cdot \mathrm{N}_{\mathrm{A}}$, where $\rho_{\mathrm{i}}$ is its scattering length density, $d$ is the layer thickness, $\phi_{\mathrm{i}}$ is its volume fraction in the layer, $b_{i}$ is its scattering length and $N_{A}$ is Avogadro's number. In this region of the data, it is the product $\rho_{\mathrm{i}} . d$ that principally determines $\Gamma$, and sensitivity to the values of $\rho_{\mathrm{i}}$ or $d$ individually is poor as the effects of the two variables are coupled (i.e. the parameters are mathematically correlated in any model). As such, structural information in this $Q_{z}$-range is lacking, and its determination requires acquisition of data to mid-to-high $Q_{z}$ values (e.g. $0.05-0.3 \AA^{-1}$ ). In this case, there is greater individual sensitivity to $\rho_{\mathrm{i}}$ and $d$, although these measurements take longer as $R$ typically goes down to a level of parts per million. Measurements in other isotopic contrasts of the subphase such as pure $\mathrm{D}_{2} \mathrm{O}$ are usually performed during structural studies as this reduces uncertainties in the resolved structural parameters. Measurements in other $\mathrm{D}_{2} \mathrm{O} / \mathrm{H}_{2} \mathrm{O}$ mixtures can also be used to match in scattering length density specific components in mixtures, although such examples are relatively uncommon. It is also in this $Q_{z}$-range that increased interfacial roughness reduces the specular reflectivity. Lastly, if there are repeating structures normal to the interface, these can manifest themselves in a Bragg diffraction peak in the data, which provides added richness to the application of the technique. 
It should be pointed out that NR as a whole provides even more information than specular NR in the form of off-specular scattering (where the reflection angle is not equal to the incidence angle) [29] as well as grazing incidence small-angle neutron scattering (where a pencil beam is used to resolve lateral structures present in the plane of the interface) [30]. There are also various examples in the literature where kinetic processes have been monitored at the solid/water interface using specular NR [31-34]. The description of these types of studies is, however, outside the scope of the present review article.

\section{Early kinetic studies}

For a couple of decades after the conception of the technique, neutron reflectivity measurements of adsorbed and spread layers at the air/water interface in soft matter and biophysics became more and more common. The majority of the data were recorded on the leading SURF reflectometer at the ISIS pulsed neutron source [35], and studies typically involved measurement of the surface excess, composition and structure of the interfacial material. On the other hand, kinetic studies remained rare as the measurements were limited in neutron flux to provide fast data acquisitions, and pulsed neutron sources are generally not as stable as reactor sources, which meant that any experiment has potential for gaps to appear in time-resolved data sets.

Even so, some notable kinetic studies were published from the instrument in the period 2006-2010. These included investigations by Edler and co-workers on mesostructured polymer/surfactant films that form spontaneously at the air/water interface in work related to materials chemistry. They used scans over a mid-to-high $Q_{z}$-range with a data acquisition time of 15 min per measurement to follow the changes in shape, position and intensity of a Bragg diffraction peak in the neutron reflectivity profiles, which afforded information about the structural progression of the films. They investigated film formation as a function of the surfactant composition in mixtures involving the weak polyelectrolyte poly(ethylene imine) and concluded that at least $20 \%$ cationic surfactant in the mixture is required to promote film formation [36]. They went on to use the methodology to prepare mesoporous silica films through incorporation of tetramethoxysilane into the film matrix and concluded that dried films retain their ordered mesostructure after surfactant removal [37].

Studies were also conducted by King and Thompson on the oxidation of surfactant and lipid monolayers at the air/water interface by $\mathrm{O}_{3}$ in work related to the environment and health. A midto-high $Q_{z}$-range was again used even though only the surface excess was resolved. The measurement times were 5-20 min, and some gaps in the data (perhaps related to instability of the neutron source) were evident. They found that around $50 \%$ of the organic material remains at the interface following oxidation of oleic acid by gas-phase $\mathrm{O}_{3}$, which led to conclusions about the extent of surface activity of the reaction products [38]. They went on to show that $\mathrm{O}_{3}$ cleaves the double bond of the oleoyl strand of 1-palmitoyl-2-oleoyl-sn-glycero-3-phosphocholine (POPC), and concluded that the resulting $\mathrm{OH}$ radicals can attack the saturated palmitoyl strands of the lipid [39].

These publications demonstrated real progress in the use of specular NR to contribute to kinetic studies at the air/water interface. However, it was clear that a substantial technical development to improve the time-resolution and beam stability would be required to take the capabilities to a superior level.

\section{$\underline{4 .}$ Recent technical development}

Although the original significant works involving specular NR at the air/water interface had been carried out using the CRISP and SURF reflectometers at the ISIS pulsed neutron source, the most 
intense source of neutrons in the world is located at the Institut Laue-Langevin (ILL) in France. Historically it did not have a neutron reflectometer suitable for the study of horizontal liquid surfaces. This situation changed, however, in $\mathbf{2 0 0 9}$ with the commissioning of the Fluid Interfaces Grazing Angles ReflectOmeter (FIGARO) [40].

Various design characteristics of the FIGARO instrument make it very well suited to the study of fast processes at the air/water interface. First, it is situated on a stable reactor source, which means that time-resolved data can be acquired reliably. Second, it has a choice of chopper pairs that allows selection of high flux options at the expense of resolution. Third, it has mirrors with different maximum wavelength cut-offs that allow selection of high flux options at the expense of broader $Q_{z}$ range. Fourth, it has a low natural incidence angle (prior to any reflection using a supermirror), which means that the maximum possible neutron flux can be directed at the sample at low $Q_{z}$ values. Figure 1 shows a schematic of the primary components of the instrument.

The combined attributes of the instrument design meant that back in 2009, FIGARO had real potential to be a significant technical development in establishment of specular NR as a valuable tool in the study of kinetic and dynamic processes at the air/water interface. Indeed in the paper that summarised its technical capabilities that was published shortly afterwards, an example of a measurement at the air/water interface was included where, instead of minutes, the fitted data were acquired in just $1 \mathrm{~s}$. This demonstration hinted at the exciting scientific advances that lay ahead.

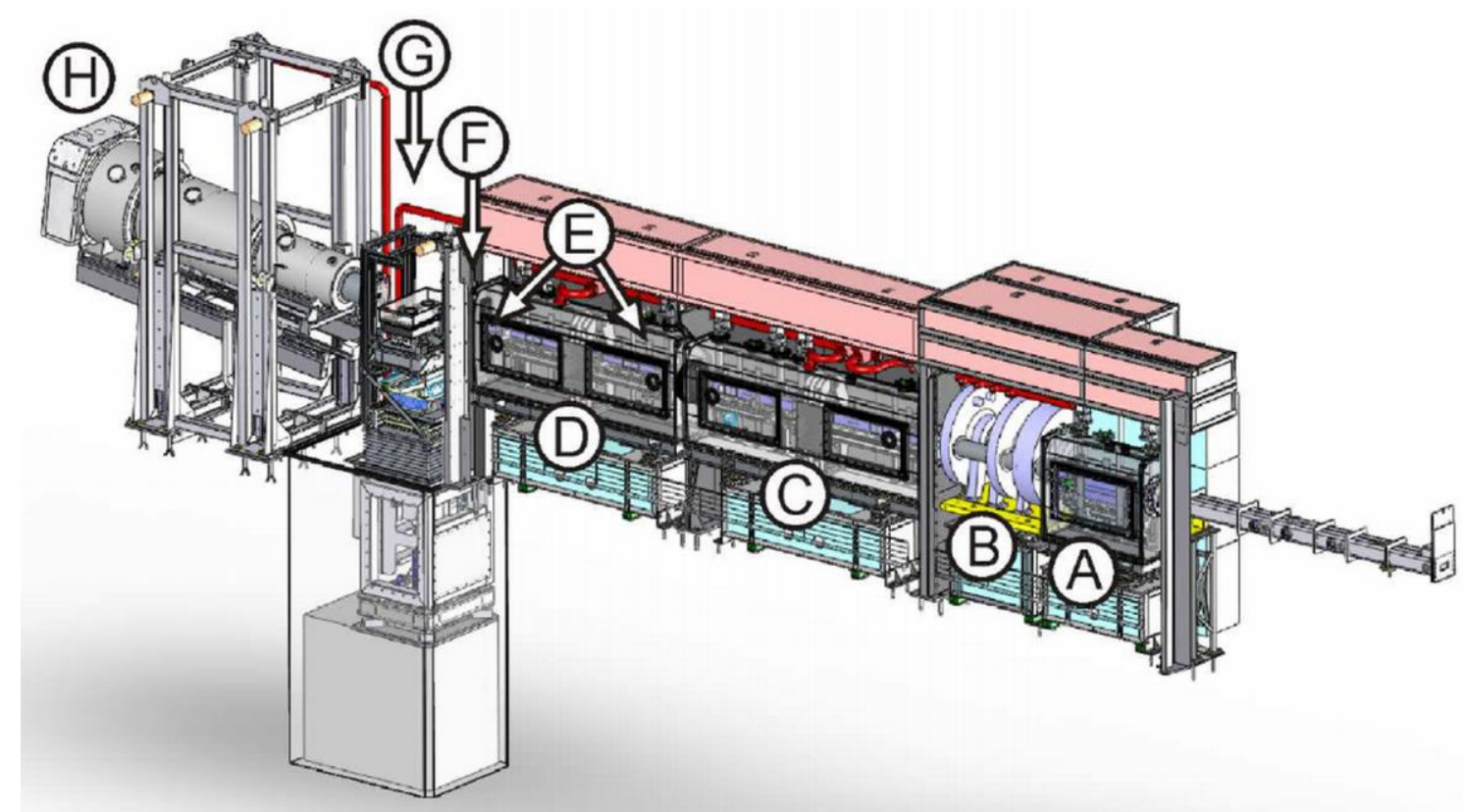

Fig. 1. Schematic of the FIGARO instrument highlighting the following components: (A) frame overlap mirrors, (B) choppers, (C) deflector mirrors, (D) collimation guide, (E) collimation slits, (F) beam attenuator, (G) sample position and $(H)$ area detector. Image is reproduced from ref. 40.

In the next few years, two different methods were developed using the FIGARO instrument to quantify the interfacial composition of binary mixtures at the air/water interface for systems where only one component is available in its deuterated form. Both methods are based on the assumption that the relationships between the surface excess and both the scattering of neutrons and the phase shift of elliptically polarised light upon reflection are additive for multiple components. It should be 
pointed out that while both methods are discussed below in the context of polymer/surfactant mixtures, they are applicable to various other mixtures, e.g., those involving lipids, proteins and drugs.

The first method was used only seldomly but involves measurement of a polymer with deuterated surfactant in ACMW at low $Q_{z}$ values using specular NR and the same mixture (albeit normally with hydrogenous surfactant in $\mathrm{H}_{2} \mathrm{O}$ for economic reasons) using ellipsometry. The measured neutron scattering excess and optical phase shift are then used in simultaneous equations to solve the surface excesses of both components. A more detailed explanation of the approach can be found in ref. [41].

The second method has had a much more significant impact and involves parallel measurements of a polymer both with deuterated surfactant in ACMW and with air contrast matched surfactant in ACMW both at low $Q_{z}$ values using specular NR. The two measured scattering excesses are then used in simultaneous equations to solve the surface excesses of both components. The first examples of use of this new 'low $Q_{z}$ interfacial composition method' can be found in refs [42]-[44] but the most detailed explanations can be found in refs [5] and [45]. Its significance merits further discussion. Traditionally, determination of the interfacial composition of a polymer/surfactant mixture using specular NR would involve the acquisition of data in multiple isotopic contrasts over the full $Q_{z^{-}}$ range. Even on leading instruments, this may take a couple of hours in total as the values of $R$ go down to parts per million in different data sets before the background level is reached. Furthermore, determination of the interfacial composition requires the application of a detailed structural model in which a priori the layer roughness values are not known. This aspect can reduce the accuracy of the quantification. Figure 2A shows an example of such data.
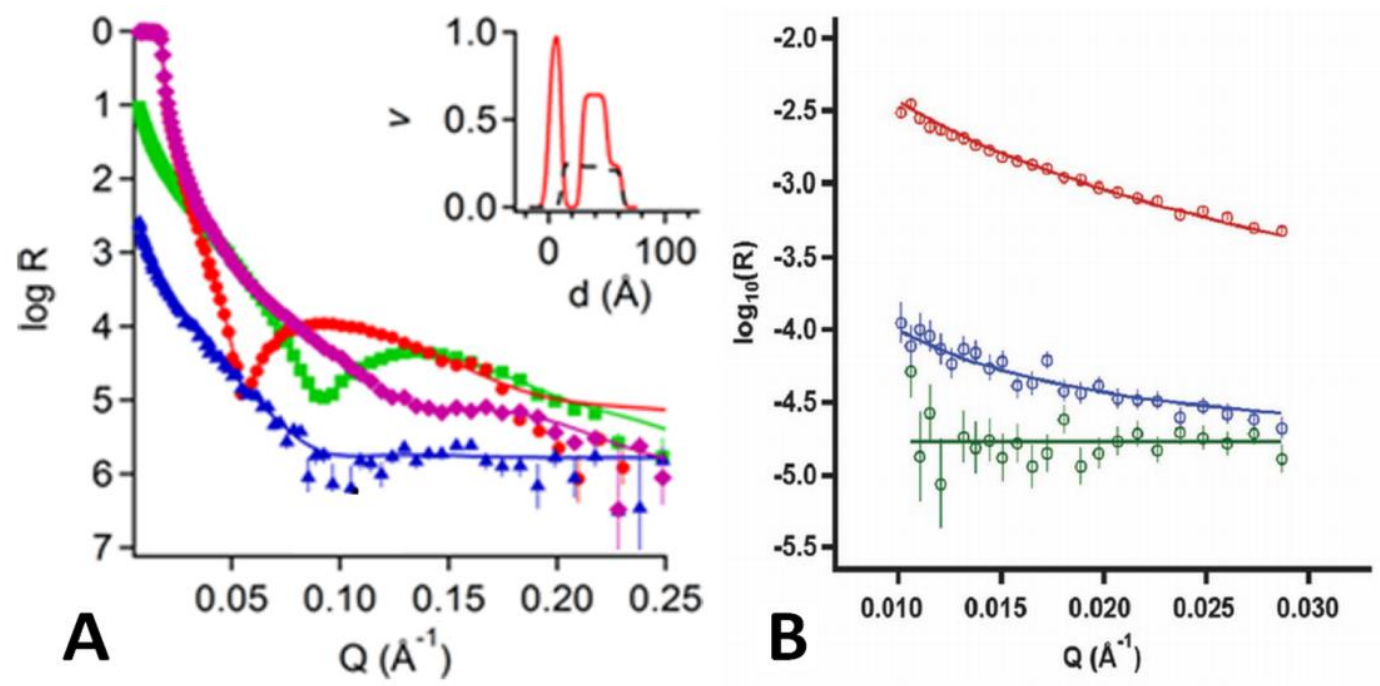

Fig. 2. (A) Specular neutron reflectivity profiles of dendrimer/surfactant mixtures recorded in multiple isotopic contrasts over a broad $Q_{z}$-range with the volume fraction profiles of the dendrimer (black dashed) and surfactant (red solid) normal to the interface shown in the inset. Image is adapted from ref. [46], and additional information about the data and samples can be found therein. (B) Specular neutron reflectivity profiles of a different polyelectrolyte/surfactant mixtures at the air/ACMW interface involving deuterated surfactant (red) and air contrast matched surfactant (blue); a background measurement is shown for reference (green). Image is reproduced from ref. [45], and additional information about the data and samples can be found therein. 
Data for the new low $Q_{z}$ interfacial composition method, in contrast, can be recorded in just a couple of minutes each, and the resulting quantified surface excesses are highly insensitive to the layer roughness values used in the model. This approach therefore offers the possibility to quantify the interfacial composition of binary mixtures more accurately and much faster than was previously possible, albeit with the loss of the structural information about the location of the components normal to the interface. Figure $2 \mathrm{~B}$ shows an example of such data. Examples of this new approach as well as its significance and impact are discussed below.

\section{Resulting scientific advances}

\subsection{Surface excess in single-component systems}

The use of high flux configurations at low $Q_{z}$ of the FIGARO instrument (e.g. a wide chopper pair and restricted $Q_{z}$-range on a stable continuous neutron source) has resulted in two key advances in the study of single-component monolayer systems: measurements of weakly reflecting monolayers and very fast measurements of deuterated monolayers.

First, it would be advantageous in general to have available all molecules such as proteins and cyclodextrins in their deuterated form. Such availability would, for example, increase the reflectivity signal of monolayers of these species above the background of ACMW in measurements conducted over a mid-to-high $Q_{z}$-range. However, custom deuteration remains expense and labour-intensive. Low- $Q_{z}$ measurements have been successfully carried out nevertheless on adsorbed layers of hydrogenous human serum albumin [47] and $\alpha$-cyclodextrin [48] in ACMW. In the former case, specular NR measurements were used as a calibration to validate the use of ellipsometry to investigate spread protein films. In the latter case, specular NR measurements were used alongside data from numerous other techniques to relate the adsorption of aggregates at the air/water interface to their size.

Second, the time resolution to follow changes in the surface excess of a deuterated monolayer at the air/water interface has been cut dramatically from minutes to seconds. Following publication of a fitted reflectivity profile recorded in $1 \mathrm{~s}$ in the paper that described the technical capabilities of the FIGARO instrument [40], Pfrang and co-workers went on to exploit this capability to provide insight into the oxidation kinetics of surfactant monolayers at the air/water interface. They started with an investigation of the oxidation of methyl oleate by gas-phase $\mathrm{O}_{3}$ and concluded that the material has an atmospheric lifetime of $10 \mathrm{~min}$, and that $<2 \%$ of it remains at the interface at the end of the reaction [49]. The data acquisition times were 1-30 s per measurement, which was tuned according to the surface excess, i.e., 1-3 s at the start of the reaction when there was high coverage and 20-30 $s$ towards the end of the reaction when there was low coverage. Figure 3 shows an example of the kinetic data that were recorded in this work.

In spite of this progress, the liquid trough had been contained in a rather large gas chamber where the gas diffusion rate was the limiting factor in the accessible oxidation rates. In response to this limitation, a miniaturised gas chamber with one thirtieth of the volume, in which it was shown that the rate coefficient could be better constrained, was designed and commissioned [50]. In this work, the potential of ellipsometry to act as a cheaper and more accessible substitute to specular NR for the study of single-component systems was evaluated. While good agreement between the data recorded using the two techniques was achieved, a degree of caution was urged as a result of the presence of temporal fluctuations in the data that were attributed to islands of material, and it was advised that each new system should be assessed on a case-by-case basis. 


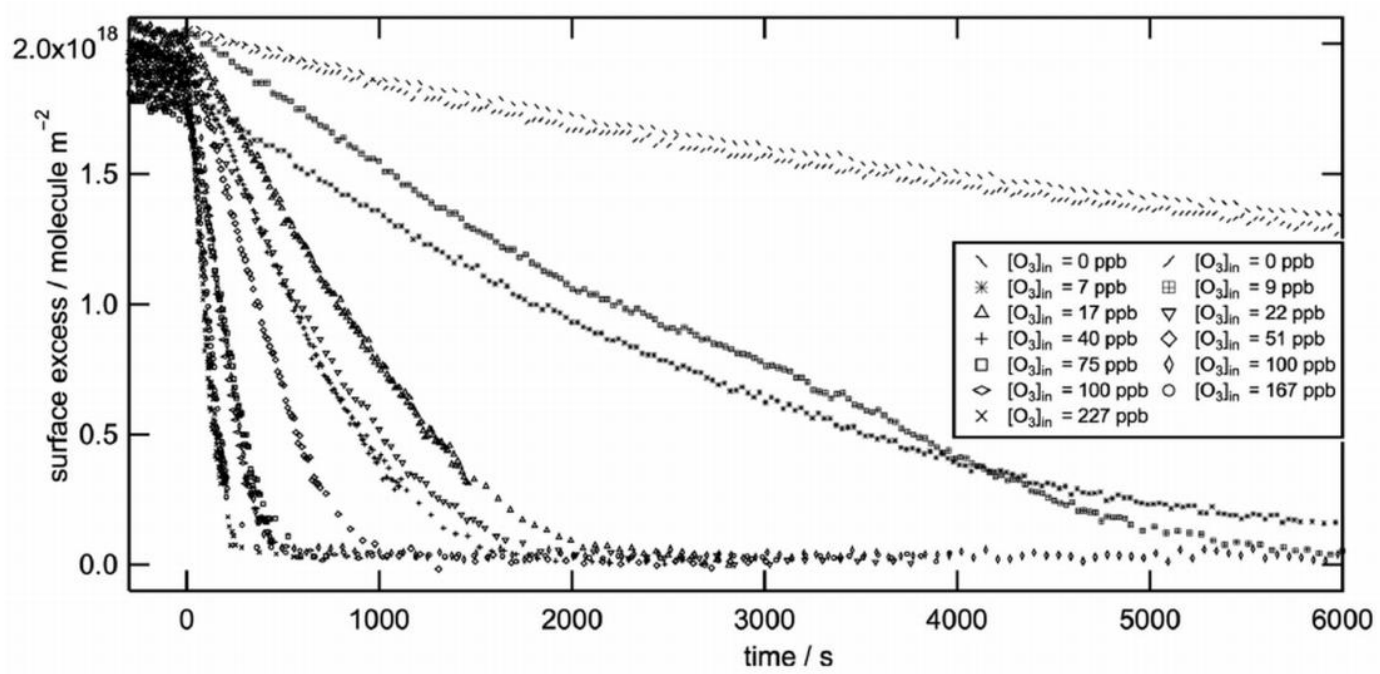

Fig. 3. Surface excess of monolayers of deuterated methyl oleate on ACMW during oxidation by $\mathrm{O}_{3}$ with respect to the reaction time and $\mathrm{O}_{3}$ concentration measured using specular NR. In situ $\mathrm{O}_{3}$ generation was initiated at $\mathrm{t}=0$. Image is reproduced from ref. 49 , and additional information about the data and samples can be found therein.

An additional combination of techniques was developed on the INTER reflectometer at the ISIS pulsed neutron source where specular NR was performed in combination with in situ ER-FTIRS [13]. This combination of techniques was applied to different monolayers system, including palmitic acid alone and mixtures of oleic acid and stearic acid. This work, in which it was demonstrated that the complementary information from ER-FTIRS provided key additional insight, marked an important step in increasing the complexity of monolayer studies of environmental relevance using specular NR.

So far the project has culminated in an extensive study, again using the FIGARO instrument, of the oxidation of four different types of surfactant monolayers by $\mathrm{NO}_{3}$, which is a gas of potential relevance to important night-time atmospheric processes [51]. In this work, the effects of chain length and degree of saturation as well as the headgroup type of the surfactant were systematically studied, and insight into the relative lifetimes of the different surfactants as well as the film forming potential of their reaction products was uniquely provided. This work represents the most comprehensive kinetic study at the air/water interface to be published using specular NR to date.

The low- $Q_{z}$ capability of FIGARO has also been used in the study of adsorption kinetics from bulk solutions in samples of hydrogenous and deuterated nanogels comprising $\mathrm{N}$-isopropylacrylamide with different amounts of the cross-linker $N, N^{\prime}$-methylenebisacrylamide [52]. The data acquisition times were 5 min per measurement. Zarbakhsh and co-workers showed that the adsorption kinetics are slower when the amount of cross-linker in the nanogel is increased, which was related to the rate of conformational changes for particles with different rigidity. Also, differences in the adsorption behaviour of the hydrogenous and deuterated samples were resolved, which was related to varying degrees of hydration of particles with different bonding energies.

In summary, exploitation of the low- $Q_{z}$ capability of the FIGARO instrument in particular has opened up studies on weakly reflecting samples in which the requirement for time-consuming and expensive custom deuteration is alleviated and, combined with parallel advances in sample environment and measurements using complementary techniques, has enabled the first extensive kinetic studies of deuterated monolayers on the second time scale. 


\subsection{Interfacial composition in binary mixtures}

The first dynamic measurements of the interfacial composition of polymer/surfactant mixtures at the air/water interface involving specular NR were performed on the continuously expanding surface of an overflowing cylinder by Bain and co-workers. The combined specular NR/ellipsometry approach described in section 4 was developed to quantify the surface excesses of both components with respect to the type and bulk composition of the mixtures. Work started on poly(ethylene oxide)/surfactant mixtures involving either tetradecyltrimethylammonium bromide or sodium dodecyl sulfate [41]. It was shown that adsorption of polymer is under diffusion control while that of surfactant is under mixed kinetic/diffusion control at low bulk surfactant concentrations, and inhibition of polymer adsorption at high bulk surfactant concentrations was different for the two systems. Work progressed to focus on an investigation of the interfacial interactions of bulk aggregates in mixtures of poly(ethylene imine) with sodium dodecyl sulfate [53]. A case was demonstrated where surprisingly the majority of the material at the dynamic air/water interface was delivered by the dissociation and Marangoni spreading from aggregates rather than the diffusion and adsorption of bulk complexes, which has been considered to be generally responsible for the delivery of interfacial material in such systems.

An approach used to provide insight into the adsorption of only one component in a binary mixture involves neglect of the scattering from the polyelectrolyte in polyelectrolyte/surfactant mixtures as a result of its minimal scattering length density. Different groups used this method to good effect by performing time-resolved measurements on mixtures involving poly(diallyldimethylammonium chloride). Varga and co-workers used the method to demonstrate the nature of trapped films out of equilibrium conditions [54], and Guzmán and co-workers went on to study the adsorption kinetics of concentrated mixtures [55]. In the latter case, the project concerned the study of samples that are closer in composition to those used in real commercial formulations. The results were modelled by an adsorption-equilibration of the interfacial layers as a two-step process involving the adsorption of complexes followed by their reorganization at the interface. The work is likely to lead on to additional progress on mixtures that are more relevant to their applications. Figure 4 shows an example of the kinetic data that were recorded in this work.

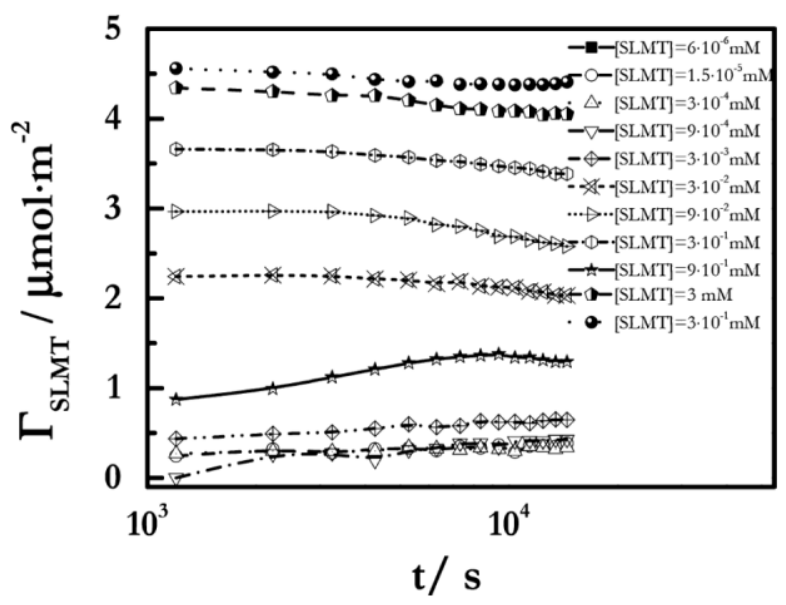

Fig. 4. Dynamic surfactant surface excesses for the adsorption of polyelectrolyte/surfactant mixtures with different bulk concentrations of the surfactant (SLMT) at the air/water interface measured using specular NR. Lines are guides for the eyes. Image is reproduced from ref. 55, and additional information about the data and samples can be found therein. 
It was work by Tummino and co-workers that showcased the new low $Q_{z}$ interfacial composition method of FIGARO during dynamic compression/expansion cycles of a surface film for the first time [45]. The principle was to form trapped polyelectrolyte/surfactant films by exploiting the dissociation and Marangoni spreading of liquid crystalline aggregates as they contacted a bare water surface combined with the retention of material at the interface due to the entropy associated with counterion release to the bulk. The interfacial composition resolved during compression/expansion cycles of the films obeyed a strict 1:1 charge binding and the films were shown to be insoluble over successive cycles. It was demonstrated in this work that the new low- $Q_{z}$ approach is more accurate and much faster than was previously possible for a binary mixture involving only one deuterated component. The work propagated to provide a systematic characterisation of films prepared from aggregates with respect to their charge/structure and the ionic strength of the subphase [56]. Here it was shown that it is possible to control the formation of extended structures either by successive spreading or compression of the films but only when the aggregates used in their preparation have a certain charge/structure. It was commented that while extended structures at the air/water interface have been observed in various works, this was the first time that control has been gained over their extent, composition and morphology. Figure 5 shows an example of the dynamic data recorded in this work.
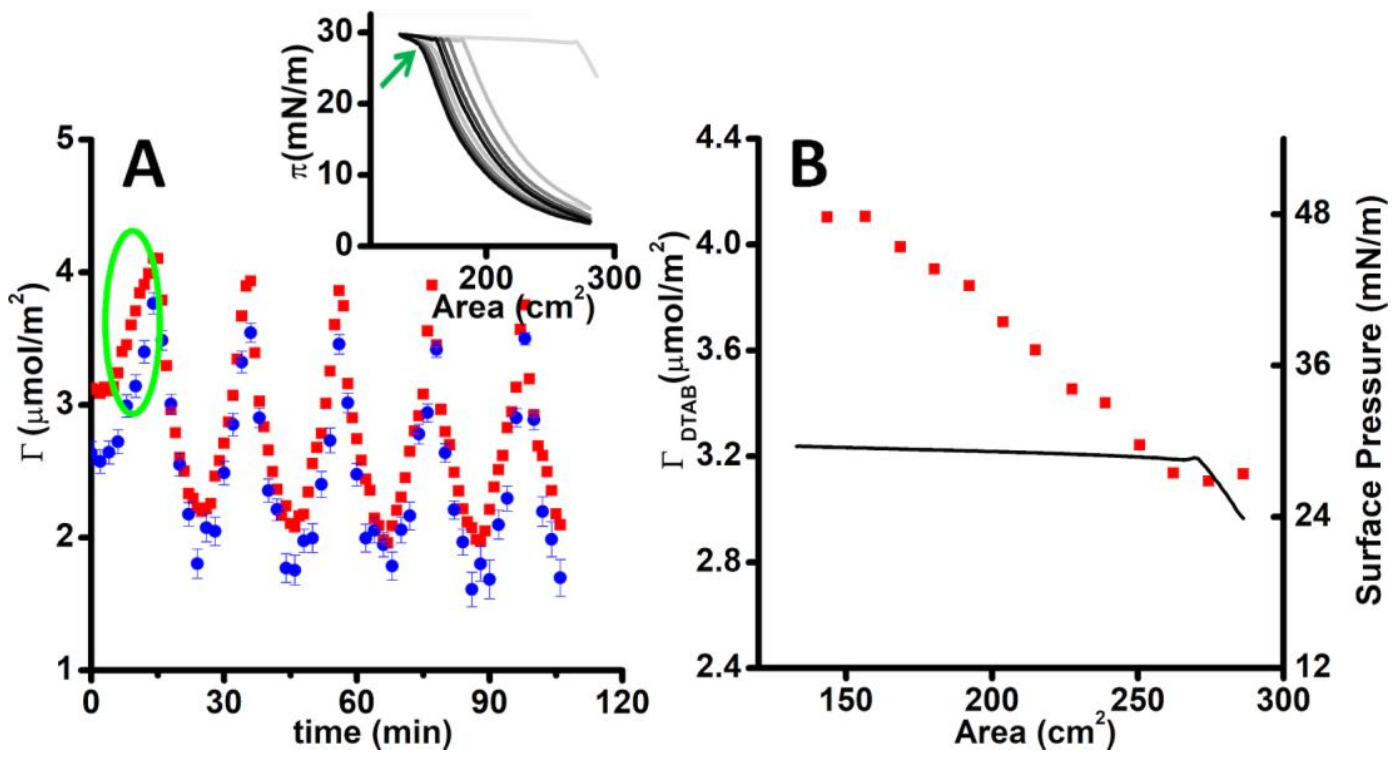

Fig. 5. (A) Interfacial composition of polyelectrolyte (blue circles) and surfactant (red squares) in films at the air/water interface spread from liquid crystalline aggregates measured using specular NR. Corresponding surface pressure data are shown in the inset. (B) Plot of the surfactant surface excess (red squares) and surface pressure (black line) during the first compression. Image is reproduced from ref. 56, and additional information about the data and samples can be found therein.

Lastly, the first time that the low $Q_{z}$ interfacial composition method of FIGARO was used to monitor the kinetics of adsorption at the air/water interface was in the work of Lu and co-workers on the interaction of short designed antimicrobial peptides with phospholipid monolayers. The interfacial composition of both components during the interaction was resolved in real time and it was shown that the peptide bound 4 times more to an anionic lipid monolayer than to a zwitterionic lipid monolayer [57]. The project extended to demonstrate that the kinetics and extent of interaction of the peptide with anionic lipid monolayers were greater at higher surface pressures [58]. Together 
the studies provided insight into the disruptive nature of antimicrobial peptides on model lipid membranes. Figure 6 shows an example of the kinetic data recorded in this work.

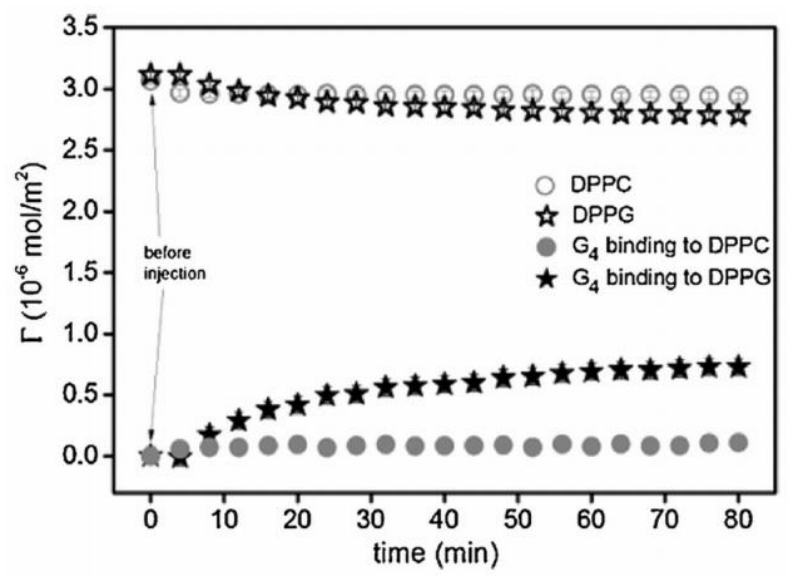

Fig. 6. Interfacial composition as a function of time for the interaction of an antimicrobial peptide (G4) with different phospholipid monolayers at the air/water interface measured using specular NR. Image is reproduced from ref. 57, and additional information about the data and samples can be found therein.

\subsection{Interaction mechanisms}

In contrast to the environmental interests in the study of oxidation kinetics of surfactant monolayers at the air/water interface by gas phase ozone discussed above, the focus of Thompson and coworkers in analogous studies involving lipid monolayers was oxidation mechanisms of relevance to health. They conducted work on the mechanism of oxidation and fate of the reaction products of different components of lung surfactant: the unsaturated lipid POPC as well as peptide sequences of lung surfactant protein B.

In the former study, the approach was to acquire time-resolved data during the oxidation of two different partially deuterated analogues of the lipid, one with the saturated chain deuterated and the other only with the terminal C9 portion of unsaturated chain deuterated [59]. It was shown that the terminal C9 portion of the unsaturated chain is lost from the interface during oxidation, and this is accompanied by an increase in the surface pressure. These observations were attributed to a reorientation of the lipid molecules that remain at the interface. It was also shown that there is no loss of saturated lipid upon exposure to ozone in its mixtures with POPC, and so the possibility of its attack by $\mathrm{OH}$ radicals generated as a result of the oxidation of the unsaturated lipid could be discounted. In the latter study, it was shown that the peptides remain at the interface following oxidation with little change in their hydration in spite of a significant resulting change in the surface pressure [60].

These time-resolved studies demonstrate the importance of partial deuteration and smart experimental design in solving interaction mechanisms in mixed systems of biophysical relevance at the air/water interface.

\subsection{Interfacial structures}

Lastly there have been some individual pieces of work that involved time-resolved measurements of evolving interfacial structures. For example, Müller-Buschbaum and co-workers investigated the drying process of solution-cast statistical copolymer films [61]. They monitored changes in the shape of the critical edge of total reflection of polymer blends in deuterated toluene using low- $Q_{z}$ data 
acquisitions. They concluded that the maximum evaporation velocity and the time taken to reach equilibrium both depend on the molecular weight of the polymer.

Time-resolved structural studies have also been conducted by exploiting measurements over a broader $Q_{z}$-range. For example, Edler and co-workers built on their previous work on mesostructured polymer/surfactant films to perform a study using several reflectometry techniques [62]. In combination with ellipsometry and Brewster angle microscopy measurements, they showed that the maximum size of a Bragg diffraction peak from specular NR is related to a growth-collapse mechanism: first the film gets thicker driven by an evaporative steady state, and then $\mathrm{CO}_{2}$ adsorption causes its breakup. Also, Noskov and co-workers recently exploited protein deuteration to resolve information about the adsorption kinetics, structure and morphology of lysozyme monolayers at the air/water interface in the absence and presence of high bulk concentrations of strong denaturants [63]. They showed that the denaturants result in an acceleration of adsorption and an increase of the adsorption layer thickness, which together were attributed to incomplete collapse of the globules in the bulk. These studies demonstrate that, although they are not as fast as low- $Q_{z}$ measurements, kinetic studies involving measurements across a mid-to-high $Q_{z}$-range have great value in resolving structural information about various interfacial phenomena in soft matter studies.

\section{Summary and outlook}

In the last few years, specular neutron reflectometry has started to provide a significant contribution in resolving information about kinetic and dynamic processes at the air/water interface in systems relevant to soft matter, biophysics, environment and health. The reason for this step change in the capabilities of the technique is primarily the commissioning of the FIGARO instrument at the ILL in 2009 with its high flux options at low $Q_{z}$ values. Uniquely, the instrument has been used to determine the surface excess of deuterated single-component systems on the second time scale (previously minutes) and the interfacial composition of binary mixtures in which only one component is deuterated on the minute time scale (previously typically more than an hour for a full characterisation).

In this review article, the current capabilities of the technique have been described, followed a systematic description of the primary recent advances. The vast majority (although not all) of the examples discussed were performed on FIGARO, which underlines its significant impact in this field. For single-component monolayers, information about weakly reflecting, non-deuterated samples and much faster kinetic information about deuterated monolayers is now routinely determined. A particular highlight was systematic work on the kinetic analysis of a selection of different deuterated surfactant monolayers, which provided information about the atmospheric lifetimes and fate of reaction products from oxidation reactions. Different methods of providing compositional information about binary mixtures involving only one deuterated component were then described. The most significant one is application of the low- $Q_{z}$ interfacial composition method involving measurements of 2 samples in ACMW where the interfacial components have different isotopic contrast. This advance has led to provision of the interfacial composition during dynamic compression/expansion cycles of films on a Langmuir trough for the first time. A particular highlight in this case was work on the nature of polyelectrolyte/surfactant films spread from aggregates in which it was shown that use of aggregates of a certain charge/structure can result in control over the formation of extended structures. Time-resolved data have also been acquired to provide information about the interaction mechanism of $\mathrm{O}_{3}$ with phospholipids thanks to their partial 
deuteration, and on the structural progression of a number of different systems involving polymers, surfactants and proteins.

As a result of the technical developments described in the present review, the low- $Q_{z}$ interfacial composition method is becoming quickly established as a key tool in the provision of kinetic and dynamic information about systems at the air/water interface in soft matter and biophysics. This information was simply not accessible previously. Scientists in Germany, for example, have resolved the kinetics of interaction of hexane vapour with different surfactant monolayers [64]. Scientists in France have investigated kinetic effects of fluorocarbon vapour on protein/phospholipid mixtures at the air/water interface [65]. Scientists in Sweden have studied the interaction dynamics of an anaesthetic drug with phospholipid monolayers during compression/expansion cycles on a Langmuir trough [66]. Also, scientists in Poland have examined interactions of an anti-cancer drug using an analogous approach [67]. A stream of further publications in the coming years is therefore anticipated as a result of this new methodology.

Further to the recent advances described, the future holds great promise in the further development of the field. There are three approaches that have started to be tested to increase the available flux on the FIGARO instrument. The first concerns operation of its choppers with a large opening angle instead of a small one. This change can result in several times the flux at the expense of resolution, as it is highly likely that the methods to resolve the surface excess and interfacial composition using data only at low $Q_{z}$ can cope with a significant compromise in the resolution. The second concerns the use of a much more divergent beam for flat samples without off-specular scattering while using the area detector to resolve the incidence angle [68]. The third concerns stopping the choppers altogether and using a prism instead to resolve the wavelength on the area detector, which is a technique called Refractive Analysis of the Incoming Neutron Beam Over the White Spectrum (RAINBOWS) $[69,70]$. A combination of these approaches could result in an increase in flux of much more than an order of magnitude, which will clearly open up faster kinetic and dynamic studies on the instrument. As we look further to the future, the FREIA instrument at the European Spallation Source, which is under construction, promises an increase in flux of much more than an order of magnitude as well.

With faster instrument capabilities in the near future, increased possibilities to resolve faster processes will arrive. It would be pertinent for us to bear in mind, however, the lesson learned concerning the reaction kinetics work involving the large gas chamber containing a Langmuir trough. Following the technical development of the FIGARO instrument, it was quickly evident that the sample environment represented the limiting factor in actual exploitation of the high neutron flux as a result of the slow gas diffusion rate. Therefore with the future technical developments that have the potential to impact this field significantly, it will be essential that the significant investment in instrumentation is matched with an appropriate investment in state-of-the-art sample environments to ensure that the new capabilities can be fully exploited.

\section{Acknowledgements}

The author thanks Karen Edler as well as the following instrument scientists for helpful discussions during the preparation of this review article: John Ankner of the Spallation Neutron Source (United States of America), Thomas Arnold of the European Spallation Source (Sweden), Mario Campana and Maximilian Skoda of the ISIS Pulsed Neutron Source (United Kingdom), Fabrice Cousin of the Laboratoire Léon Brillouin (France) and Andrew Nelson of the Australian Centre for Neutron Scattering (Australia). 


\section{$\underline{\text { References }}$}

1. Lu JR, Thomas RK, Penfold J. Surfactant layers at the air/water interface: structure and composition. Adv Colloid Interface Sci 2000;84:143-304.

2. Penfold J, Ward RC, Williams WG. A time-of-flight neutron reflectometer for surface and interfacial studies. J Phys E 1987;20;1411-7.

3. Sivia D. Elementary scattering theory: for x-ray and neutron users. Oxford University Press; 2011.

4. Narayanan $\mathrm{T}$, Wacklin $\mathrm{H}$, Konovalov $\mathrm{O}$, Lund R. Recent applications of synchrotron radiation and neutrons in the study of soft matter. Crysallogr Rev 2017;23:160-226.

5. Braun L, Uhlig M, von Klitzing R, Campbell RA, Polymers and surfactant at fluid interfaces studied with specular neutron reflectometry. Adv Colloid Interface Sci 2017;247:130-48.

6. Yaseen A, Wang Y, Su TJ, Lu JR. Surface adsorption of zwitterionic surfactants: $n$-alkyl phosphocholines characterised by surface tensiometry and neutron reflection. J Colloid Interface Sci 2005;288:361-70.

7. Knyght I, Clifton L, Saaka Y, Lawrence MJ, Barlow D. Interaction of the antimicrobial peptides Rhesus $\theta$ defensin and porcine protegrin-1 with anionic phospholipid monolayers. Langmuir 2016;32:7403-10.

8. Lee L-T, Jha BK, Malmsten M, Holmberg K. Lipase-surfactant interactions studied by neutron reflectivity and ellipsometry. J Phys Chem B 1999;103:7489-94.

9. Aguié-Béghin V, Baumberger S, Monties B, Douillard R. Formation and characterization of spread lignin layers at the air/water interface. Langmuir 2002;18:5190-6.

10. Foglia F, Fragneto G, Clifton LA, Lawrence MJ, Barlow DJ. Interaction of amphotericin B with lipid monolayers. Langmuir 2014;30:9147-56.

11. Sun HH, Resmini M, Zarbakhsh A. Interaction of thermal responsive NIPAM nanogels with model lipid monolayers at the air-water interface. J Colloid Interface Sci 2018;518:97-106.

12. Weygand $M$, Schalke $M$, Howes PB, Kjaer K, Friedmann J, Wetzer B, Pum D, Sleytr UB, Lösche M. Coupling of protein sheet crystals (S-layers) to phospholipid monolayers. J Mater Chem 2000;10:141-8.

13. Skoda MWA, Thomas B, Hagreen M, Sebastiani F, Pfrang C. Simultaneous neutron reflectometry and infrared reflection absorption spectroscopy (IRRAS) study of mixed monolayer reactions at the air-water interface. RSC Advances 2017;7:34208-14.

14. Dabkowska A, Talbot JP, Cavalcanti L, Webster JRP, Nelson A, Barlow DJ, Fragneto G, Lawrence MJ. Calcium mediated interaction of calf-thymus DNA with monolayers of distearoylphospharidylcholine: a neutron and X-ray reflectivity study. Soft Matter 2013;9:7095-105.

15. Hazell G, Gee AP, Arnold T, Edler KJ, Lewis SE. Langmuir monolayers composed of single and double tail sulfobetaine lipids. J Colloid Interface Sci 2016;474:190-8.

16. Anderson PM, Wilson MR. Molecular dynamics simulations of amphiphilic graft copolymer molecules at the water/air interface. J Chem Phys 2004;121:8503.

17. Xue Y, He L, Middleberg APJ, Mark AE, Poger D. Determining the structure of interfacial peptide films: comparing neutron reflectometry and molecular dynamics simulations. Langmuir 2014;30:10080-9.

18. Wierenga PA, Gruppen H. New views on foams from protein solutions. Curr Opin Colloid Interface Sci 2010;15:365-73.

19. Liu F, Wang ZN, Wang DZ, Wei XL, Zhou W, Li GZ, Zhang GY. Adsorption kinetics of Brij 97 at the air/solution interface. J Dispersion Sci Technol 2006;27:657-63. 
20. Xu L, Han GB, Hu JW, He Y, Pan JG, Li YJ, Xiang JN. Hydrophobic coating- and surface active solventmediated self-assembly of charged gold and silver nanoparticles at water-air and water-oil interfaces. Phys Chem Chem Phys 2009;11:6490-7.

21. Lopes-Costa T, Gámez F, Lago S, Pedrosa JM. Adsorption of DNA to octadecylamine monolayers at the air-water interface. J Colloid Interface Sci 2011;354:733-8.

22. Mmereki BT, Donaldson DJ, Gilman JB, Eliason TL, Vaida V. Kinetics and products of the reaction of gas-phase ozone with anthracene adsorbed at the air-aqueous interface. Atmos Environ 2004;38:6091103.

23. Firooz A, Chen P. Surface tension and adsorption kinetics of amphiphiles in aqueous solutions: The role of carbon chain length and temperature. J Colloid Interface Sci 2012;370:183-91.

24. Reeser DI, Jammoul A, Clifford D, Brigante M, D'Anna B, George C, Donaldson DJ. Photoenhanced reaction of ozone with chlorophyll at the seawater surface. J Phys Chem C 2009;113:2071-7.

25. Guzmán E, Liggieri L, Santini E, Ferrari M, Ravera F. Effect of hydrophilic and hydrophobic nanoparticles on the surface pressure response of DPPC monolayers. J Phys Chem C 2011;115:21715-22.

26. Noskov, BA, Krycki MM. Dilational surface rheology of polymer and polymer/surfactant solutions. Adv Colloid Interface Sci 2010;15:229-36.

27. Bain $C D$, Manning-Benson S, Darton RC. Rates of mass transfer and adsorption of hexadecyltrimethylammonium bromide at an expanding air-water interface. J Colloid Interface Sci 2000;229:247-56.

28. Leckner J. Theory of reflection. Vol. 87 of Springer series on atomic, optical, and plasma physics. Cham: Springer International Publishing; 2016.

29. Pynn R, Baker S, Smith G, Fitzsimmons M. Off-specular scattering in neutron reflectometry. J Neutron Res 1999;7:139-58.

30. Müller-Buschbaum P, Gutmann JS, Cubitt R, Stamm M. Probing the in-plane composition of thin polymer films with grazing-incidence small-angle neutron scattering and atomic force microscopy. Colloid Polym Sci 1999;1199:1193-9.

31. Jouault N, Corvis Y, Cousin F, Jestin J, Barre L. Asphaltene adsorption mechanisms on the local scale probed by neutron reflectivity: transition from monolayer to multilayer growth above the flocculation threshold. Langmuir 2009;25:3991-8.

32. Zhong Q, Metwalli E, Kaune G, Rawolle M, Bivigou-Koumba AM, Laschewsky A, Papadakis CM, Cubitt $R$, Müller-Buschbaum P. Switching kinetics of thin thermos-responsive hydrogel films of poly(monomethoxy-diethyeleneglycol-acrylate) probed with in situ neutron reflectivity. Soft Matter 2012;8:5241-9.

33. Gerelli Y, Porcar L, Lombardi L, Fragneto G. Lipid exchange and flip-flop in solid supported bilayers. Langmuir 2013;29:12762-9.

34. Koutsioubas A, Appavou M-S, Lairez D. Time-resolved neutron reflectivity during supported membrane formation by vesicle fusion. Langmuir 2017;33:10598-605.

35. Penfold J, Richardson RM, Zarbakhsh A, Webster JRP, Bucknall DG, Rennier AR, Jones RAL, Cosgrove T, Thomas RK, Higgins JS, Fletcher DPI, Dickinson E, Roser SJ, McLure IA, Hillman AR, Richards RW, Staples EJ, Burgess AN, Simister EA, White JW. Recent advances in the study of chemical surfaces and interfaces by specular neutron reflection. J Chem Soc Faraday Trans 1997;93:3899-3917. 
36. O'Driscoll BMD, Fernandez-Martin C, Wilson RD, Roser SJ, Edler KJ. Effect of micelle composition on the formation of surfactant-templated polymer films. J Phys Chem B 2006;110:5330-6.

37. Yang B, Edler KJ. Free-standing ordered mesoporous silica films synthesized with surfactantpolyelectrolyte complexes at the air/water interface. Chem Mater 2009;21:1221-31.

38. King MD, Rennie AR, Thompson KC, Fisher FN, Dong CC, Thomas RK, Pfrang C, Hughes AV. Oxidation of oleic acid at the air-water interface and its potential effects on cloud critical supersaturations. Phys Chem Chem Phys 2009;11:7699-7707.

39. Thompson KC, Rennie AR, King MD, Hardman SJO, Lucas COM, Pfrang C, Hughes BR, Hughes AV. Reaction of a phospholipid monolayer with gas-phase ozone at the air-water interface: measurement of surface excess and surface pressure in real time. Langmuir 2010;26:17295-303.

**40. Campbell RA, Wacklin HP, Sutton I, Cubitt R, Fragneto G. FIGARO: the new horizontal neutron reflectometer at the ILL. Eur Phys J Plus 2011;126:107.

$\rightarrow$ Highlight. Commissioning of the FIGARO instrument at the Institut Laue-Langevin has led to the vast majority of the novel studies of fast kinetic and dynamic processes discussed in this review article - its situation on a stable reactor source makes the instrument suited to time-resolved studies while other important attributes include its low natural incidence angle and high flux configurations.

41. Angus-Smyth A, Campbell RA, Bain CD. Dynamic adsorption of weakly interacting polymer/surfactant mixtures at the air/water interface. Langmuir 2012;28:12479-92.

*42. Ábraham Á, Campbell RA, Varga I. New method to predict the surface tension of complex synthetic and biological polyelectrolyte/surfactant mixtures. Langmuir 2013;29:11554-9.

$\rightarrow$ Highlight. This was the paper where the physical basis was outlined for the first time of the low- $Q_{z}$ interfacial composition method on FIGARO involving measurement of data in 2 isotopic contrasts that are both in ACMW including one that is very weakly reflecting - although only one set of data was presented in the Supporting Information with this work the approach was born.

43. Ábrahám Á, Kardos A, Mezei A, Campbell RA, Varga I. Effects of ionic strength on the surface tension and nonequilibrium interfacial characteristics of poly(sodium styrene sulfonate)/ dodecyltrimethylammonium bromide mixtures. Langmuir 2014;30:4970-9.

44. Fauser $\mathrm{H}$, von Klitzing $\mathrm{R}$, Campbell RA. Surface adsorption of oppositely charged $\mathrm{C}_{14}$ TAB-PAMPS mixtures at the air/water interface and the impact on foam film stability. J Phys Chem B 2015;119:34858.

**45. Campbell RA, Tummino A, Noskov BA. Varga I. Polyelectrolyte/surfactant films spread from neutral aggregates. Soft Matter 2016; 12:5304-12.

$\rightarrow$ Highlight. The low- $Q_{z}$ interfacial composition method for dynamic measurements of binary mixtures at the air/water interface during compression/expansion cycles on a Langmuir trough was highlighted in this work - it was demonstrated that the method is more accurate and much quicker than was previously possible to achieve using the traditional broad $Q_{z}$-range approach.

46. Yanez Arteta M, Campbell RA, Watkins E, Obiols-Rabasa M, Schillén K, Nylander T. Interactions of small dendrimers with sodium dodecyl sulfate at the air/water interface. J Phys Chem B 2014;118:1183548.

47. Campbell RA, Ang JC, Sebastiani F, Tummino A, White JW. Spread films of human serum albumin at the air-water interface: optimization, morphology, and durability. Langmuir 2015;31:13535-42. 
48. Hernandez-Pascacios J, Piñeiro Á, Ruso JM, Hassan N, Campbell RA, Campos-Terán J, Costas M. Complex behavior of aqueous $\alpha$-cyclodextrin solutions. Interfacial morphologies resulting from bulk aggregation. Langmuir 2016;32:6682-90.

**49. Pfrang C, Sebastiani F, Lucas COM, King MD, Hoare ID, Chang D, Campbell RA. Ozonoysis of methyl oleate monolayers at the air-water interface: oxidation kinetics, reaction products and atmospheric implications. Phys Chem Chem Phys 2014;16:13220-8.

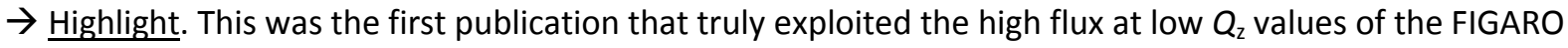
instrument to deliver a full research study using surface excess measurements on the second time scale this work provided insight into the atmospheric lifetime and fate of reaction products from the oxidation reaction of ozone with surface monolayers at the air/water interface.

50. Sebastiani F, Campbell RA, Pfrang C. Complementarity of neutron reflectometry and ellipsometry for the study of atmospheric reactions at the air-water interface. RSC Advances 2015;5:107105-11.

*51. Sebastiani F, Campbell RA, Rastogi K, Pfrang C. Night-time oxidation of surfactants at the air-water interface: effects of chain length, head group type and saturation degree. Atmos Chem Phys 2018;18:3249-68.

$\rightarrow$ Highlight. This kinetic study at the air/water interface is comprehensive and provides atmospheric insight with respect to several physical variables - effects of surfactant chain length, charge and degree of saturation on the oxidation of monolayers at the air/water interface by $\mathrm{NO}_{3}$ were systematically investigated using a new miniaturised sample environment dedicated for the purpose.

52. Zielinska K, Sun H, Campbell RA, Zarbakhsh A, Resmini M. Smart nanogels at the air/water interface. Nanoscale 2016;8:4951-60.

53. Angus-Smyth A, Bain CD, Varga I, Campbell RA. Effects of bulk aggregation on PEI/SDS monolayers at the dynamic air/liquid interface: depletion due to precipitation versus enrichment by a convection/spreading mechanism. Soft Matter 2013;9:6103-17.

54. Varga I, Campbell RA. General physical description of the behavior of oppositely charged polyelectrolyte/surfactant mixtures at the air/water interface. Langmuir 2017;33:5915-24.

*55. Llamas S, Guzmán E, Akanno A, Fernández-Peña L, Ortega F, Campbell RA, Miller R, Rubio RG. Study of the liquid / vapor interfacial properties of concentrated polyelectrolyte - surfactant mixtures using surface tensiometry and neutron reflectometry: equilibrium, adsorption kinetics and dilational rheology. J Phys Chem C 2018;122:4419-27.

$\rightarrow$ Highlight. This paper focuses on adsorption kinetics and responses to dynamic perturbations of the surface area of polyelectrolyte/surfactant layers in concentrated mixtures through quantification of the surfactant surface excess during adsorption - the work represents an important step to access mixtures that are closer in composition to the formulations used in real consumer products.

*56. Tummino A, Toscano J, Sebastiani F, Noskov BA, Varga I, Campbell RA. Effects of aggregate charge and subphase ionic strength on the properties of spread polyelectrolyte/surfactant films at the air/water interface under static and dynamic conditions. Langmuir 2018;34:2312-23.

$\rightarrow$ Highlight. The low $-Q_{z}$ interfacial composition method was used to reveal that the formation of extended structures in polyelectrolyte/surfactant films could be switched on or off according to the type of aggregates used in their preparation - control over the amount, composition and morphology of extended structures at the air/water interface was demonstrated for the first time. 
**57. Ciumac D, Campbell RA, Xu H, Clifton LA, Hughes AV, Webster JRP, Lu JR. Implications of lipid monolayer charge characteristics on their selective interactions with a short antimicrobial peptide. Colloids Surf B 2017;150:308-16.

$\rightarrow$ Highlight. The low- $Q_{z}$ interfacial composition method was used to quantify the kinetics of penetration of a short designed antimicrobial peptide into phospholipid monolayers at the air/water interface for the first time - lipid lost from the interface as a result was quantified too and the extent of the interaction was attributed to the favourability of electrostatic interactions.

58. Ciumac D, Campbell RA, Clifton LA, Xu H, Lu JR. Influence of acyl chain saturation on the membrane binding activity of a short designed antimicrobial peptide. ACS Omega 2017;2:7482-92.

59. Thompson KC, Jones SH, Rennie AR, Ward AD, Hughes BR, Lucas COM, Campbell RA, Hughes AV, King $M D$. Degradation and rearrangement of a lung surfactant lipid at the air-water interface during exposure to the pollutant gas ozone. Langmuir 2013;29:4594-602.

60. Hemming JM, Hughes BR, Rennie AR, Tomas S, Campbell RA, Hughes AV, Arnold T, Botchway SW, Thompson KC. Environmental pollutant ozone causes damage to lung surfactant protein B, SP-B. Biochemistry 2015;54:5185-97.

61. Diethert A, Metwalli E, Meier R, Zhong Q, Campbell RA, Cubitt R, Müller-Buschbaum P. In-situ neutron reflectometry study of the near-surface solvent concentration profile during solution casting. Soft Matter 2011;7:6648-59.

62. Campbell RA, Edler KJ. Growth-collapse mechanism of PEI-CTAB films at the air-water interface. Soft Matter 2011;7:11125-32.

63. Campbell RA, Tummino A, Varga I, Milyaeva OY, Krycki MM, Lin S-Y, Laux V, Haertlein M, Forsyth VT, Noskov BA. Adsorption of denaturated lysozyme at the air-water interface: structure and morphology. Langmuir 2018, 34, 5020-5029.

64. Pradines V, Fainerman VB, Aksenenko EV, Kragel J, Mucic N, Miller R. Adsorption of alkyl trimethylammonium bromides at the water/air and water/hexane interfaces. Colloids Surf $A$ 2010;371:22-8.

65. Nguyen PN, Waton G, Vandamme T, Krafft MP. Reversing the course of the competitive adsorption between a phospholipid and albumin at an air-water interface. Soft Matter 2013;9:9972-6.

66. Niga P, Hansson-Mille PM, Swerin A, Claesson PM, Schoelkopf J, Gane PAC, Bergendal E, Tummino A, Campbell RA, Johnson CM. Interactions between model cell membranes and the neuroactive drug propofol. J Colloid Interface Sci 2018, 526, 230-246.

67. Matyszewska D, Brzezinska K, Juhaniewicz J, Bilewicz R. pH dependence of daunorubicin interactions with model DMPC:Cholesterol membranes. Colloids Surf B 2015;134:295-303.

68. Cubitt R, Saerbeck T, Campbell RA, Barker R, Gutfreund P. An improved algorithm for reducing reflectometry data involving divergent beams or non-flat samples. J Appl Crystallog 2015;48:2006-11.

69. Cubitt R, Stahn J. Neutron reflectometry by refractive encoding. Eur Phys J Plus 2011;126:111.

70. Cubitt R, Seguera Ruiz J, Jark W. RAINBOWS: refractive analysis of the incoming neutron beam over the white spectrum. A new fast neutron reflectometry technique exploiting a focusing prism. J Appl Crystallog 2018;51:257-63. 